\title{
Alternativas de integración de competitividad regional entre los departamentos de Antioquia, Magdalena y Meta, 2017
}

Regional Competitiveness Integration Alternatives of the Departments of Antioquia, Magdalena and Meta, 2017

Jesús Rafael Fandiño Isaza

Administrador de empresas, especialista en Gerencia Estratégica de Mercadeo y magister en MBA de Negocios, Líder del grupo de investigación INCACEN y del semillero SIGMA, Docente Ocasional Tiempo Completo Universidad Nacional Abierta y a Distancia UNAD, Santa Marta, Magdalena.

Correo electrónico: jesus.fandino@unad.edu.co

\section{Albeiro Ospina Penagos}

Economista con área de especialización en Finanzas, psicólogo, y magister en MBA de Negocios, docente universitario, asesor en proyectos de desarrollo social y empresarial.

Correo electrónico: aospinape@unadvirtual.edu.co 


\section{Belma Luz Pérez Ruiz}

Administradora de empresas, aspirante maestría en Administración de Organizaciones.

Correo electrónico: blperezr@unadvirtual.edu.co

Faber Anselmo Ariza Bonilla

Ingeniero agroindustrial, especialista en Gerencia Estratégica, funcionario público, con formación técnica en gestión de la inocuidad y calidad alimentaria, asesor de empresas del sector agroalimentario, administrador de la operación catering en establecimientos de alimentos y bebidas.

Correo electrónico: faarizab@unadvirtual.edu.co

\section{Resumen}

Desde el año 2000 la CEPAL se ha encargado de realizar el escalafón de competitividad departamental en Colombia, teniendo en cuenta los cinco pilares determinantes de la competitividad propuestos por la Comisión Económica para América Latina y el Caribe (CEPAL), a saber: 1) Gobierno e instituciones; 2) Desarrollo económico; 3) Infraestructura productiva; 4) Capital humano, y 5) Eficiencia de las empresas. Adicionalmente, desde el año 2013 el Consejo Privado de Competitividad junto con el Centro de Pensamiento en Estrategias Competitivas de la Universidad del Rosario (CEPEC), realizan el Índice Departamental de Competitividad. Estos resultados se han convertido en la base del análisis de competitividad en cada uno de los departamentos, permitiendo evaluar el progreso de las diferentes variables socioeconómicas que allí se miden. El análisis sugiere que la competitividad puede adquirir más relevancia entre los departamentos al interior de pequeños grupos, por lo que se propone alternativas de Integración de Competitividad Regional de los departamentos de
Antioquia, Magdalena y Meta; pretendiendo ganar relevancia las políticas de crecimiento y desarrollo regional, con una propuesta de fortalecimiento de la infraestructura, el capital humano y procesos de innovación.

Palabras clave: competitividad, alternativas de integración regional, ventajas competitivas, eficiencia y productividad, asociatividad, factores diferenciales.

\section{Abstract}

Since 2000, ECLAC has been responsible for making the departmental competitiveness ladder in Colombia, taking into account the five pillars of competitiveness proposed by the Economic Commission for Latin America and the Caribbean (ECLAC), namely: 1) Government and institutions; 2) Economic development; 3) Productive infrastructure; 4) Human capital, and 5) Efficiency of companies. In addition, since 2013, the Private Competitiveness Council, together with the Center for Thinking on Competitive 
Strategies of the Universidad del Rosario (CEPEC), has carried out the Departmental Competitiveness Index. These results have become the basis of the analysis of competitiveness in each one of the Departments, allowing to evaluate the progress of the different socioeconomic variables that are measured there. The analysis suggests that competitiveness may acquire more relevance among the departments within small groups, for which Alternative Integration of Regional Competitiveness of the Departments of Antioquia, Magdalena and Meta is proposed; The growth and regional development policies are intended to gain relevance, with a proposal to strengthen infrastructure, human capital and innovation processes.

Keywords: Competitiveness, regional integration alternatives, competitive advantages, efficiency and productivity, associativity, differential factors.

\section{Introducción}

La competitividad se entiende como la capacidad de una organización pública o privada, que busca mantener sistemáticamente ventajas comparativas y competitivas, por lo que hace imprescindible determinar la real capacidad de las distintas regiones de un país con el fin de establecer proyectos que de alguna manera beneficien a los diferentes estamentos de la región. En este sentido, se requiere consenso para la construcción de estrategias orientadas a lograr un mayor nivel de desarrollo el que no se podría alcanzar de manera aislada.

Ante la necesidad de encontrar alternativas y soluciones a las problemáticas de un país, las regiones, se han constituido en las fuentes para impulsar el crecimiento económico local y lograr el resurgir de movimientos regionales que reclaman mayor autonomía política por lo limitado de los recursos, y con el fin de sumar sinergias, capitales, talentos, instituciones es necesario desarrollar una visión conjunta partiendo de las potencialidades y características de cada región eficientes fueron las políticas y esfuerzos realizados por las diferentes regiones, para mejorar su competitividad y ser eficientes día a día.

Con el presente artículo se buscan alternativas de unidad de las regiones y ciudades vinculadas en la investigación mediante la interacción de personas y actividades económicas en un área geográfica que destaque la Competitividad Regional entre los Departamentos de Antioquia, Magdalena y Meta en el año 2017.

\section{Materiales o recursos}

El presente estudio está orientado en una in investigación de tipo documental, en un lapso de tiempo de un año (2017).

El presente trabajo se realizó mediante la recolección, selección, análisis y presentación de información coherente a partir del uso de los documentos publicados por el Consejo Privado de Competitividad en sus informes anuales, y del Índice Departamental de Competitividad (IDC), pero fundamentados en los pilares determinantes que establece la CEPAL, en su Escalafón de la Competitividad de los Departamentos de Colombia (ECD) para el año 2017, periodo analizado para conocer los factores determinantes de la integración regional.

Para el tratamiento de la información, se utilizó el programa informático de Excel, para organizar los datos de cada departamento en estudio, evaluados anualmente por el IDC. El paso siguiente fue establecer la correspondencia entre los factores que miden el IDC y ECD (Tabla 1). 


\begin{tabular}{|l|l|}
\hline IDC & ECD \\
\hline Pilar 1. Gobierno e instituciones. & Instituciones \\
Pilar 2. Desarrollo económico & Tamaño del mercado \\
Pilar 3. Infraestructura productiva & Eficiencia de los mercados \\
Pilar 4. Capital Humano & Infraestructura \\
Pilar 5. Eficiencia de las empresas & Educación básica y media \\
& Salud \\
& Educación superior y capacitación \\
& Sostenibilidad ambiental \\
\hline
\end{tabular}

Tabla 1. Correspondencia entre IDC y ECD.

Fuente. autores.

Se procedió a la tabulación de cada uno de los pilares definidos, seleccionando la posición según el indicador del pilar respectivo para cada departamento incluido en la base de datos y se organizó la información para el año 2017, en una sola hoja de Excel, seleccionando los datos correspondientes a los departamentos Antioquia, Magdalena y Meta, para proceder a representar los resultados y comparar los valores alcanzados, respecto a los pilares del ECD, mediante la construcción de gráficos para el período de análisis, y destacar las diferencias más destacada entre estas regiones.

\section{Resultados y discusión}

\section{Ranking general}

El primer resultado analizado en la Tabla 2, corresponde a la posición que dentro del total de departamentos que se evalúan (26), los departamentos en estudio. Antioquia, está siempre dentro de los 10 primeros lugares, alcanzando su mejor ranking (2) para el año 2017, con una evaluación de 6.67. En la misma Tabla 2, se destaca que el departamento del Meta, se encuentra ubicado en la posición (11) en el año 2017, con un puntaje de 4.59.

\begin{tabular}{|l|l|l|l|}
\hline Año & Departamento & $\begin{array}{l}\text { Posición } \\
\text { (Entre 26) }\end{array}$ & $\begin{array}{l}\text { Puntaje } \\
(\mathbf{0}-10)\end{array}$ \\
\hline \multirow{3}{*}{2017} & Antioquia & 2 & 6,67 \\
\cline { 2 - 4 } & Magdalena & 18 & 4,36 \\
\cline { 2 - 4 } & Meta & 11 & 4,59 \\
\hline
\end{tabular}

Tabla 2. Ranking y valor general por año. Fuente. Consejo Privado de Competitividad \& CEPECUniversidad del Rosario (2017).

Por último, el departamento del Magdalena en el puesto 18, mejora su posición con relación al periodo anterior (puesto 22 en 2016), y en 2017 obtiene un puntaje de 4.36 . 


\section{Gobierno e instituciones}

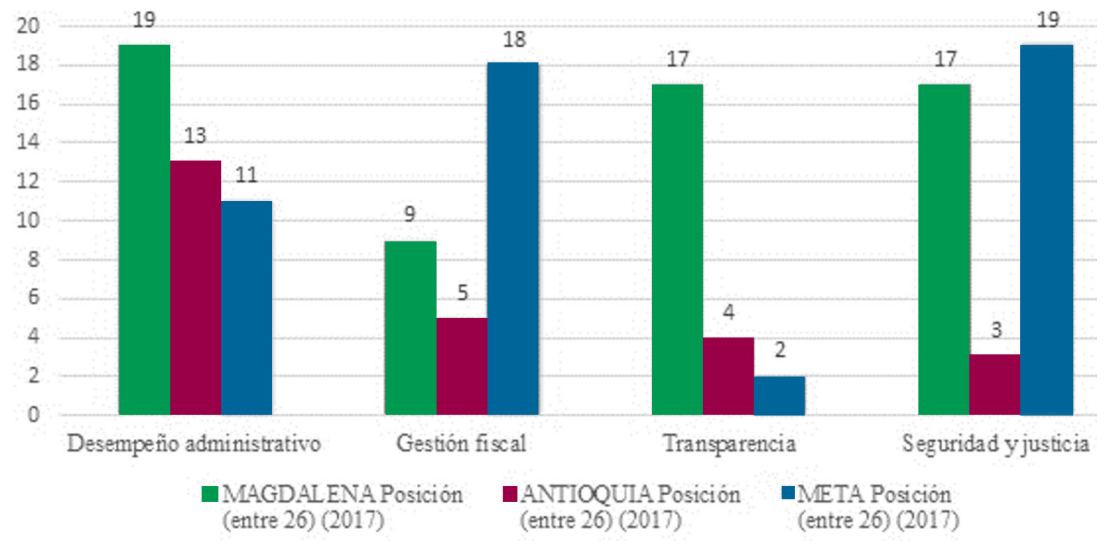

Gráfico 1. Indice comparativo instituciones departamentos de Antioquia, Magdalena y Meta. Fuente. Consejo Privado de Competitividad \& CEPEC-Universidad del Rosario (2017).

El Gráfico 1 muestra el comportamiento de las variables pilar Instituciones en un comparativo de los departamentos de Antioquia, Magdalena y Meta durante el año 2017 donde se observa que el Meta tiene un mejor desempeño administrativo que Antioquia y Magdalena. En cuanto a la Gestión Fiscal Antioquia y Magdalena superan al Meta, en el índice de transparencia, Meta y Antioquia superan al Magdalena, por último, en materia de seguridad y justicia Antioquia sobrepasa a Magdalena y Meta.

Con miras al fortalecimiento de la competitividad en una región, se requiere articular esfuerzos, las instituciones y los recursos en pos de construcción de una Agenda Regional que identifique y adopte los siguientes ejes temáticos, según la Cámara de Comercio de Medellín (2017, p. 16):

- Innovación y desarrollo tecnológico,

- Formación de talento humano,

- Infraestructura,

- Desarrollo institucional,

- Medio ambiente

- Mipymes,

- Productividad

En este sentido, en la búsqueda de un entorno de negocios que posibilite a las empresas ser más competitivo en un mundo globalizado, se ha pro- mueve la consolidación de la estrategia clúster en Medellín, Antioquia, como modelo para la construcción de redes de cooperación y colaboración entre empresas de los departamentos en estudio, con el fin de suscitar el crecimiento económico y social de estas regiones, principalmente en las industrias en que naturalmente son competitivas; resaltando la participación de todos los agentes relevantes: empresas, proveedores de servicios, academia e instituciones públicas (Cámara de Comercio de Medellín, 2016).

Otra estrategia importante es ampliar el sistema clúster, como un modelo de asociatividad de comprobados beneficios, desde la geografía económica se han reconocido las ventajas del modelo clúster; la proximidad entre las empresas y las instituciones de apoyo y los mercados son también causas del crecimiento y de los diferenciales de productividad entre países y regiones. (Cámara de Comercio de Medellin, 2009).

Los clústeres considerados en el modelo "Medellín ciudad clúster" para este estudio son clúster de los sectores construcción, turismo, medicina, y textil. En consecuencia, la política basada en clúster presenta algunas diferencias con los enfoques tradicionales basados en sectores: en primer lugar, se acentúa la relación entre los recursos que se abren paso en distintas industrias (p. ej., la rela- 
ción entre algunas especializaciones industriales o incluso agrícolas y de servicios), y en segundo lugar, el interés se centra en intensificar el uso del conocimiento y en mejorar la interacción constructiva entre las diferentes partes de la red (Cámara de Comercio de Medellín para Antioquia, s.f.).

\section{Infraestructura Productiva}

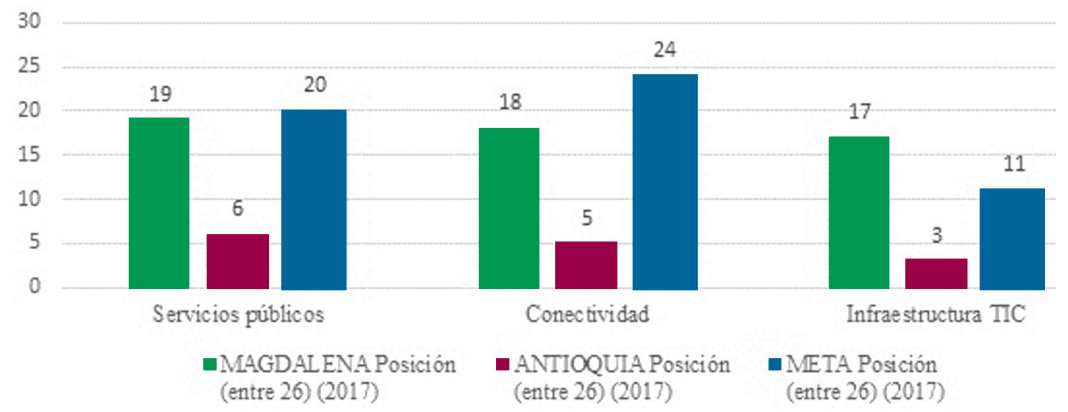

Gráfico 2. Indice comparativo infraestructura departamentos de Antioquia, Magdalena y Meta. Fuente. Consejo Privado de Competitividad \& CEPEC-Universidad del Rosario (2017).

La infraestructura productiva es también relevante para la fortaleza económica de una región, en el contexto que si el crecimiento económico es muy volátil, debido a la materialización de riesgos por perturbaciones externas e internas, cuando se presentan efectos negativos sobre la productividad, la acumulación de capital humano y la composición de la inversión (Aravena et al., 2014), porque se infiere una correlación negativa entre fortaleza económica y volatilidad del crecimiento económico, sin embargo, una economía fuerte debe ser capaz de realizar las transformaciones estructurales para maximizar la productividad y el crecimiento, a través de la reubicación de la producción en sus sectores o ramas de actividad económica. La consolidación de la industria como sector fundamental y la diversificación de la producción son estrategias de transformación estructural que permiten agregar resistencia a los mercados laborales, contribuir a la convergencia de los salarios y reducir los efectos de la volatilidad del crecimiento (Herrendorf et al., 2014).

Las grandes desigualdades entre departamentos en pilares como bienestar e infraestructura determinan el mayor peso de factores como servicios públicos, conectividad e infraestructura TIC, que se ubican como principales ventajas competitivas en las condiciones de vida, el capital humano y en la conectividad estratégica. En los últimos años gana peso la fortaleza económica y la ciencia, tecnología e innovación como factores de competitividad, lo que señala las crecientes ventajas competitivas de la diversificación productiva del departamento de Antioquia que muestra unos resultados muy positivos, superiores a los departamentos de Magdalena y Meta.

Amenazas externas, como la caída de los precios internacionales del petróleo que golpean fuertemente a los departamentos de Meta y Magdalena, motivó que ahora gane importancia la diversidad, más que el tamaño del mercado. La inseguridad, especialmente en secuestros y extorsiones, en el contexto del Acuerdo de paz, presenta una menor diferenciación regional.

El análisis de la infraestructura productiva, muestra que las políticas implementadas por el departamento de Antioquia en la prestación de servicios públicos, conectividad e infraestructura TIC, está focalizada como estrategia de competi- 
tividad y mejoramiento de la calidad de vida de sus habitantes y es prioridad en los planes de desarrollo del departamento, mientras que en el caso de Meta y Magdalena se ven como departamentos secundarios en los resultados de este factor, lo que hace que el nivel de competitividad no sea el mejor, las condiciones son tan diferentes, y, en busca de la integración regional y mejorar el desempeño en estos índices, se debe disminuir los niveles de corrupción que ostentan los departamentos de Magdalena y Meta, por debajo de Antioquia.

Por otro lado, el departamento de Antioquia ocupa el cuarto lugar en transparencia, además que agrupa otras ventajas competitivas en el país; especialmente en lo económico, ciencia, tecnología e innovación, factores con grandes brechas con los departamentos de Meta y Magdalena.

Las políticas públicas sectoriales de estos departamentos deben apuntar a estos temas estratégicos y a programas enfocados en el desarrollo de ventajas competitivas o en el cierre de estas brechas temporales o persistentes. Las inversiones públicas nacionales deben orientarse en los departamentos de Magdalena y Meta a apoyar las agendas de competitividad departamental.

\section{Capital humano}

Una de las estrategias que deben emprenderse en integración regional de los departamentos en estudio es el desarrollo y articulación del tejido social. Desde la perspectiva de la CEPAL, el capital social es el conjunto de relaciones sociales caracterizadas por actitudes de confianza y comportamientos de cooperación y reciprocidad. Se trata, pues, de un recurso de las personas, los grupos y las colectividades en sus relaciones sociales, con énfasis, a diferencia de otras acepciones del término, en las redes de asociatividad de las personas y los grupos (Atria, 2003, p. 26).

La movilización del capital social dentro de los sectores más pobres deberá ponerse en marcha, conjuntamente con un sistema económico dinámico e incluyente, además de un amplio sistema sociopolítico que sea consistente con los objetivos de inclusión. Esta estrategia deberá estar orientada al diseño de instituciones formales de asociación y participación. La capacitación de los beneficiarios para su propia gestión nunca alcanzará sus objetivos, a menos que las instituciones socioculturales informales de confianza, cooperación, liderazgo y prestigio sean reconocidas como temas de política pública (Atria, 2003, 27).

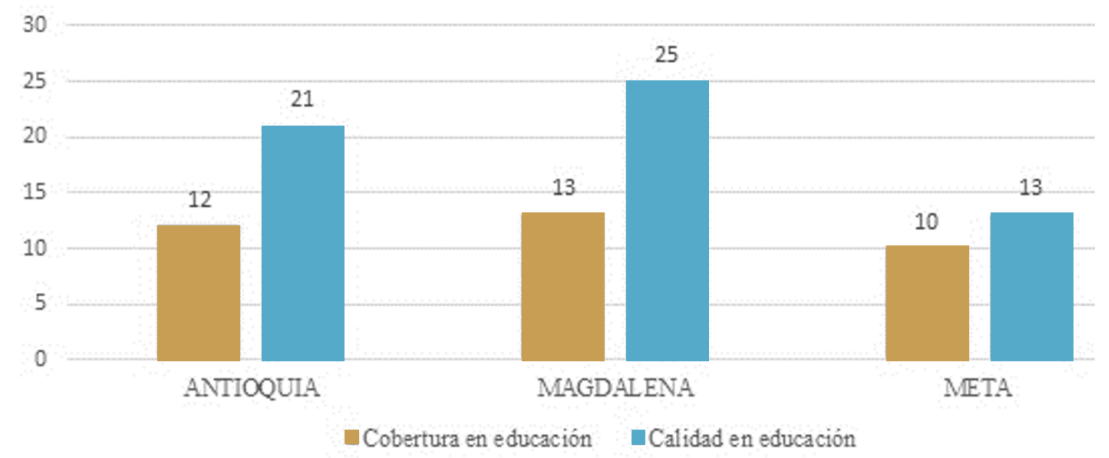

Gráfico 3. Indice comparativo educación básica y media departamentos de Antioquia, Magdalena y Meta. Fuente. Consejo Privado de Competitividad \& CEPEC-Universidad del Rosario (2017). 


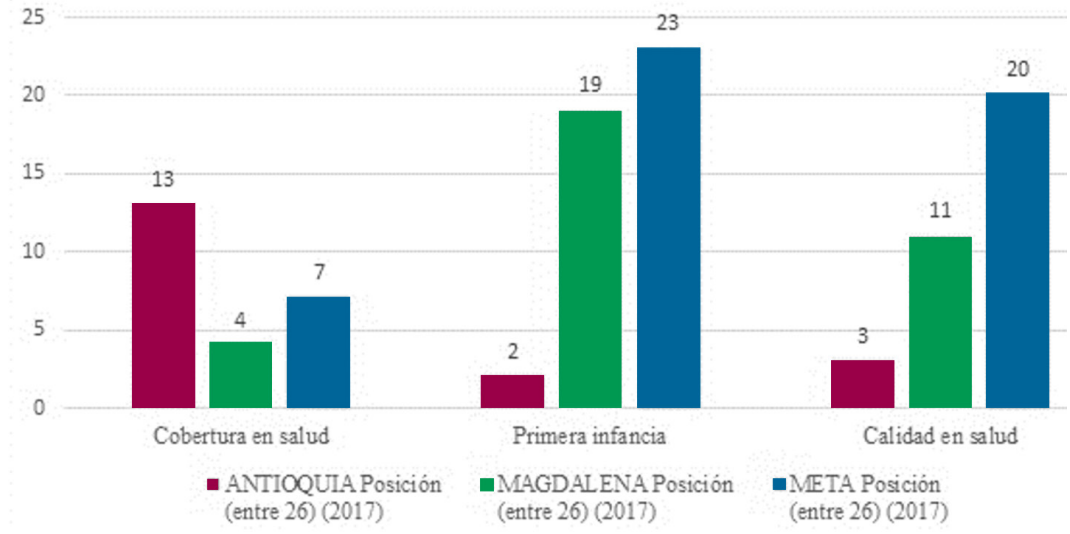

Gráfico 4. Indice comparativo salud departamentos de Antioquia, Magdalena y Meta. Fuente. Consejo Privado de Competitividad \& CEPEC-Universidad del Rosario (2017).

En el pilar de educación superior y capacitación, el departamento de Antioquia continúa en el tercer lugar del índice de competitividad. Se destaca en el subpilar de calidad en educación, fundamentalmente en las variables de calidad de docentes y cobertura de formación técnica y tecnológica, en las que ocupa el primer y tercer lugar, respectivamente. Además, este departamento ocupa el segundo lugar en dominio de segundo idioma con una calificación de 9,22 sobre 10. El departamento que más avanza en este pilar es Magdalena al escalar nueve posiciones en el IDC 2017, debido a su desempeño en la variable de cobertura en instituciones de educación superior con acreditación de alta calidad (Gráfico 5).

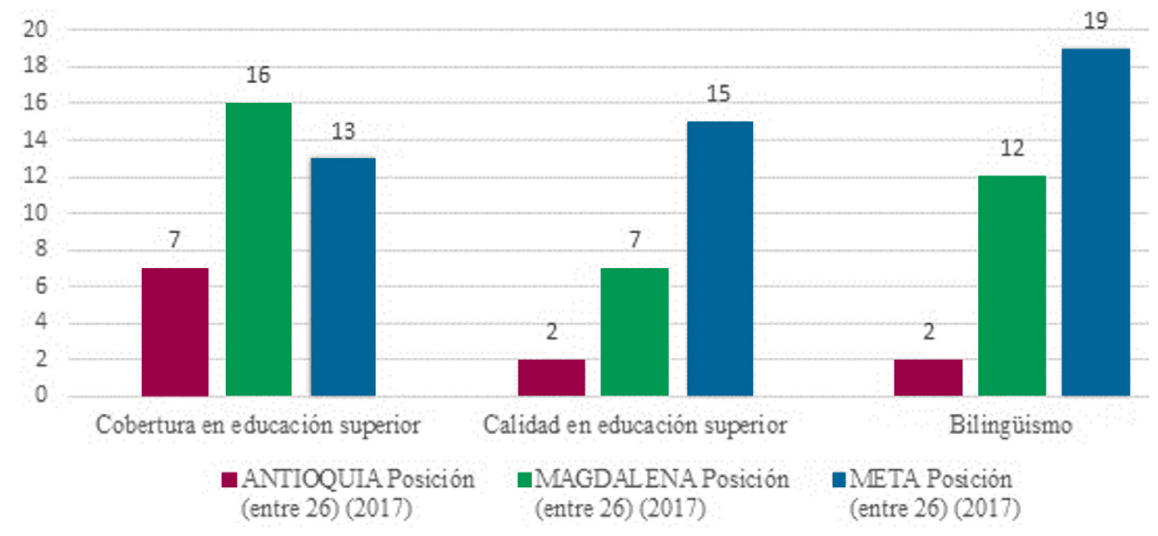

Gráfico 5. Indice comparativo educación superior y capacitación departamentos de Antioquia, Magdalena y Meta. Fuente. Consejo Privado de Competitividad \& CEPEC-Universidad del Rosario (2017). 


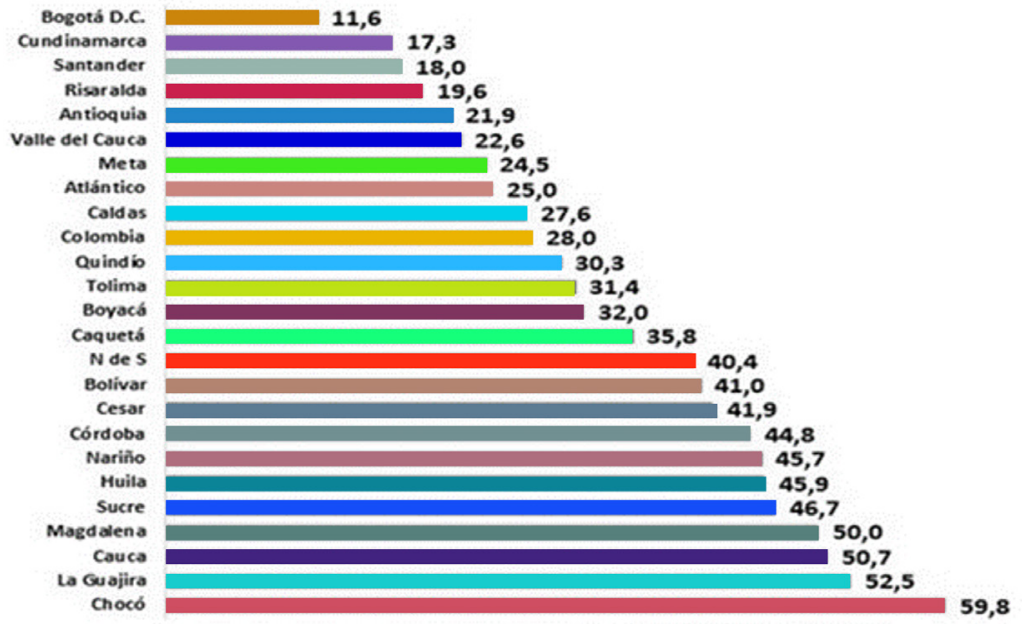

Figura 1. Pobreza moderada por departamentos (2016).

Fuente. Canavire \& Carvajal, 2017.

En el gráfico anterior, se evidencia un nivel de pobreza moderada del $21,9 \%$ en el departamento de Antioquia. La pobreza es uno de los problemas más serios Su alta incidencia indica que una gran proporción de los hogares no puede cubrir sus necesidades básicas y tampoco tiene acceso a educación, salud y bienestar lo que marca un área en la cual es básico concentrar los esfuerzos y los recursos para alivia este mal que aqueja al $20 \%$ de la población. Es por ello que es un fenómeno que requiere su estudio para determinar su tasa (can- tidad de gente en situación de pobreza), sus causas y sus efectos para poder tomar las medidas que reduzcan sus efectos y revertir su tendencia. La pobreza se caracteriza por la falta de recursos. Por lo general la pobreza se define como la falta de recursos monetarios para alcanzar un estándar mínimo de vida. Aunque los ingresos constituyen un factor fundamental para definir la pobreza, ésta abarca muchas otras dimensiones del ser y sociales (Canavire \& Carvajal, 2017).

\section{Desarrollo económico}

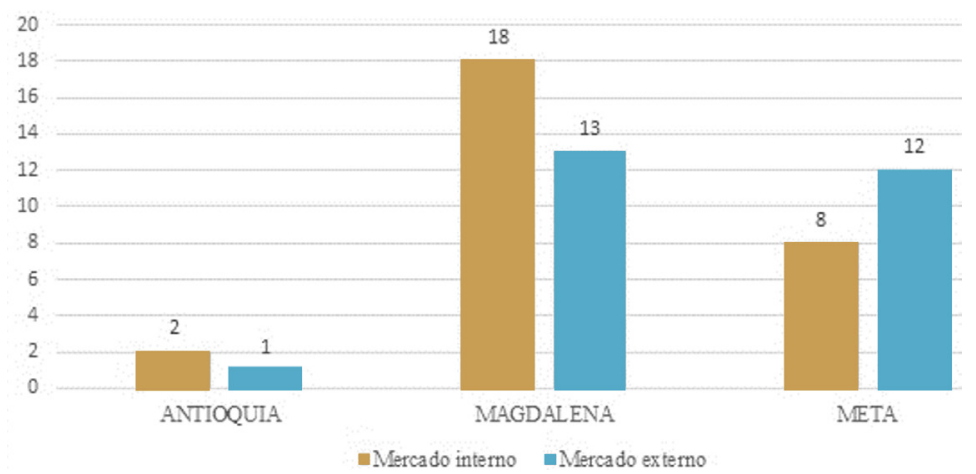

Gráfico 6. Indice comparativo tamaño de mercado departamentos de Antioquia, Magdalena y Meta. Fuente. Consejo Privado de Competitividad \& CEPEC-Universidad del Rosario (2017). 
El tamaño y crecimiento del mercado es con frecuencia el principal tema de análisis (Pike et al., 2007). Aunque el PIB per cápita y su crecimiento no son medidas completas de desarrollo económico, y de ahí la necesidad de mediciones más integrales de competitividad, están asociados con el potencial para el avance de los estándares de vida (Huggins et al., 2014).

Los escalafones internacionales de la competitividad han mostrado con suficiencia la robustez estadística de la relación entre la competitividad y la riqueza de las naciones (Herrendorf et al., 2014).

Esta conclusión se sostiene también a nivel regional; resulta natural pensar en las regiones más ricas como las más competitivas y viceversa (Martin, 2004). Pero el tamaño de los mercados tiene también una dimensión demográfica: la densidad poblacional y el nivel de urbanización regional son variables correlacionadas con la fortaleza económica. Esto es debido a que las regiones más densamente pobladas son también aquellas con mayor concentración de la actividad económica, de la demanda y del capital humano especializado.

Además, los costos de transporte disminuyen de manera sustancial con la densidad poblacional, lo que aumenta el ingreso disponible, y así la demanda por bienes y servicios del mercado. Esto a su vez se convierte en un atractivo para las empresas, que alimentan un proceso de aglomeración industrial y reducción de los precios (Morita y Yamamoto, 2013).

La estructura productiva es también relevante para la fortaleza económica. Si el crecimiento económico es muy volátil, debido a la materialización de riesgos por perturbaciones externas e internas, se presentan efectos negativos sobre la productividad, la acumulación de capital humano y la composición de la inversión (Aravena et al., 2014), y se infiere una correlación negativa entre fortaleza económica y volatilidad del crecimiento económico. Además, una economía fuerte debe ser capaz de realizar las transformaciones estructurales para maximizar la productividad y el crecimiento, a través de la reubicación de la producción en sus sectores o ramas de actividad económica.

La consolidación de la industria como sector fundamental y la diversificación de la producción son estrategias de transformación estructural que permiten agregar resistencia a los mercados laborales, contribuir a la convergencia de los salarios y reducir los efectos de la volatilidad del crecimiento (Herrendorf et al., 2014).

El comercio exterior es una porción cada vez más importante de la economía de las naciones y de las regiones, ya que provee apalancamiento para el crecimiento económico y amplía los mercados. La apertura comercial internacional contribuye a la disponibilidad y diversificación del capital, la tecnología y el trabajo especializado (Taner et al., 2000). En particular, la orientación exportadora de una economía representa el principal aprovechamiento de la apertura comercial internacional (Anderton y Dunnett, 1987).

Para el análisis del pilar Desarrollo Económico se tienen en cuenta los servicios financieros, el tamaño y crecimiento del mercado, la creación de sociedades y emprendimiento, la estructura productiva y comercio exterior. Las regiones analizadas muestran que el departamento de Antioquia potencializa con sus ventajas competitivas en la diversificación productiva y las iniciativas empresariales colocándolo en el segundo puesto a nivel nacional.

La actualización temática del factor fortaleza económica concentra el análisis en los temas económicos y de mercado. En 2017 en Colombia el departamento de Antioquia es líder en este pilar, el departamento del Meta presento un comportamiento que lo ubica en un nivel medio bajo y el departamento del Magdalena su comportamiento es de nivel bajo. 


\begin{tabular}{|c|c|c|}
\hline \multicolumn{3}{|c|}{ Departamento de Antioquia } \\
\hline & Número municipios: 125 & $\begin{array}{l}\text { Extensión territorial: } 63.612 \\
\mathrm{~km}^{2}\end{array}$ \\
\hline & $\begin{array}{l}\text { Población }(2016(\mathrm{p})) \text { : } \\
6.534 .857 \text { habitantes }\end{array}$ & $\begin{array}{l}\text { Participación en la } \\
\text { población total }(2016(p)) \text { : } \\
13,4 \%\end{array}$ \\
\hline & $\begin{array}{l}\text { Participación en el PIB } \\
\text { nacional }(2016(\mathrm{p})): 14,01 \%\end{array}$ & $\begin{array}{l}\text { PIB per cápita } 2016(\mathrm{p})^{*} \text { : } \\
\text { US } \$ 6.001\end{array}$ \\
\hline & $\begin{array}{l}\text { Exportaciones** per cápita } \\
\text { (2016): US } \$ 663.6\end{array}$ & $\begin{array}{l}\text { Importaciones*** per cápita } \\
\text { (2016): US } \$ 1.012 .5\end{array}$ \\
\hline \multicolumn{3}{|c|}{ 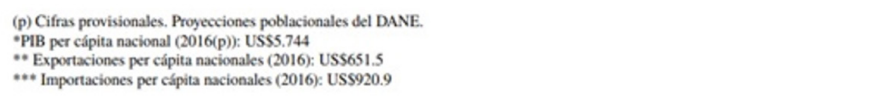 } \\
\hline \multicolumn{3}{|c|}{ Departamento de Magdalena } \\
\hline & Número municipios: 30 & $\begin{array}{l}\text { Extensión territorial: } 23.188 \\
\mathrm{~km}^{2}\end{array}$ \\
\hline & $\begin{array}{l}\text { Población }(2016(\mathrm{p})) \text { : } \\
1.272 .442 \text { habitantes }\end{array}$ & $\begin{array}{l}\text { Participación en la } \\
\text { población total }(2016(p)) \text { : } \\
2,6 \%\end{array}$ \\
\hline & $\begin{array}{l}\text { Participación en el PIB } \\
\text { nacional }(2016(p)): 1,3 \%\end{array}$ & $\begin{array}{l}\text { PIB per cápita } 2016(\mathrm{p})^{*} \text { : } \\
\text { U\$ } \$ 2.852\end{array}$ \\
\hline & $\begin{array}{l}\text { Exportaciones** per cápita } \\
\text { (2016): US } \$ 471.2\end{array}$ & $\begin{array}{l}\text { Importaciones*** per cápita } \\
\text { (2016): US } \$ 155.3\end{array}$ \\
\hline \multicolumn{3}{|c|}{ 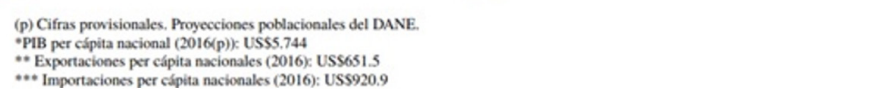 } \\
\hline \multicolumn{3}{|c|}{ Departamento de Meta } \\
\hline & Número municipios: 29 & $\begin{array}{l}\text { Extensión territorial: } 85.635 \\
\mathrm{~km}^{2}\end{array}$ \\
\hline & $\begin{array}{l}\text { Población }(2016(p)): 979.710 \\
\text { habitantes }\end{array}$ & $\begin{array}{l}\text { Participación en la } \\
\text { población total }(2016(\mathrm{p})) \text { : } \\
2 \%\end{array}$ \\
\hline & $\begin{array}{l}\text { Participación en el PIB } \\
\text { nacional }(2016(p)): 3,01 \%\end{array}$ & $\begin{array}{l}\text { PIB per cápita 2016(p)*: } \\
\text { US } \$ 8.601\end{array}$ \\
\hline$\$$ & $\begin{array}{l}\text { Exportaciones** per cápita } \\
\text { (2016): US } \$ 635.7\end{array}$ & $\begin{array}{l}\text { Importaciones*** per cápita } \\
(2016) \text { : US } \$ 40.9\end{array}$ \\
\hline
\end{tabular}

Figura 2.

Fuente. Mincomercio, 2018. 
En la compilación de datos y cifras de los departamentos analizados, muestra su desarrollo económico y participación financiera en el país, en este caso se puede inferir que el departamento de Antioquia supera significativamente en estos los factores a Meta y Magdalena, con lo que le permite ser un departamento de los más competitivos en Colombia, su concentración poblacional, participación el PIB Nacional y su participación en las exportaciones tasadas en miles de dólares.

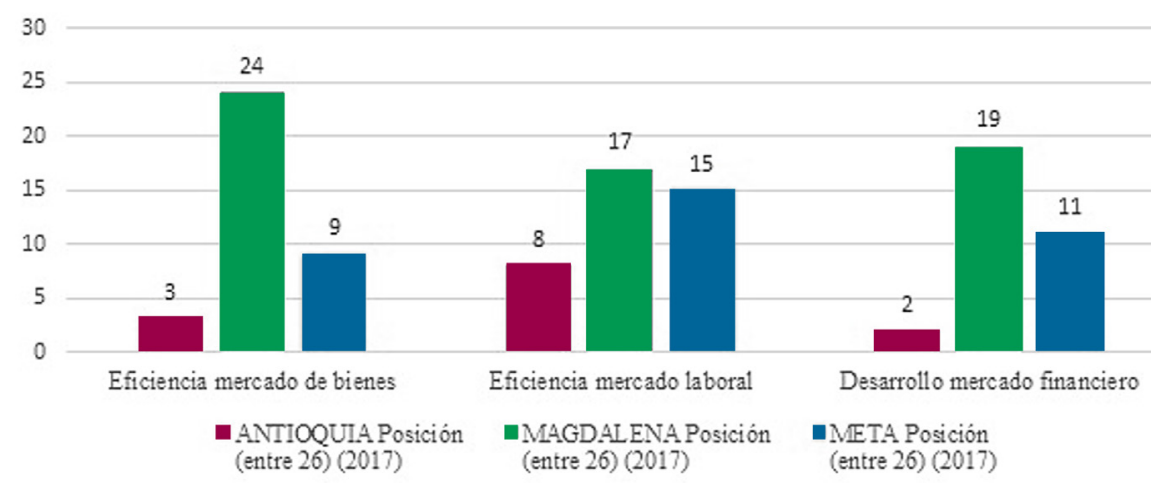

Gráfico 7. Indice comparativo eficiencia de los mercados departamentos de Antioquia, Magdalena y Meta.

Fuente. Consejo Privado de Competitividad \& CEPEC-Universidad del Rosario (2017).

\section{Eficiencia de las empresas}

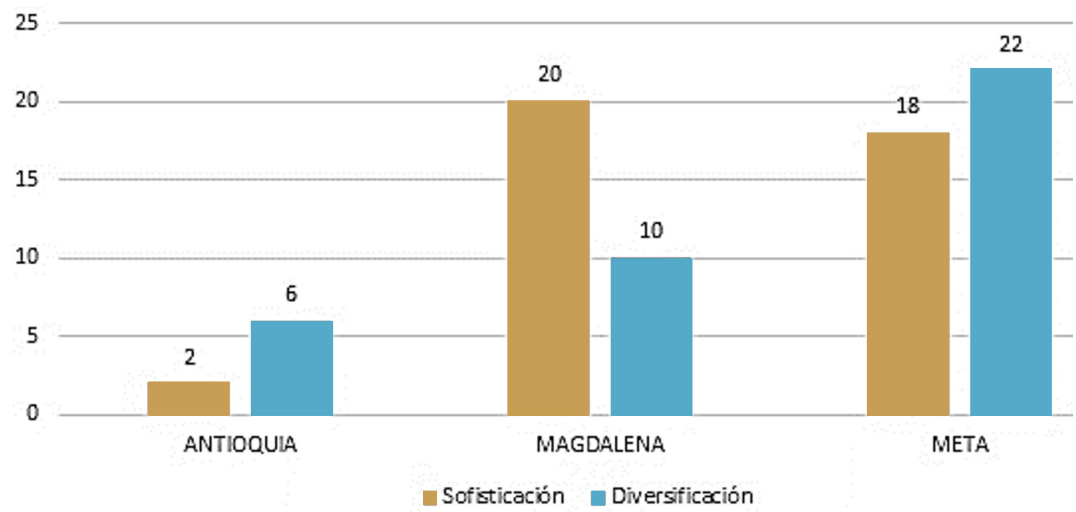

Gráfico 8. Indice comparativo sofisticación y diversificación departamentos de Antioquia, Magdalena y Meta. Fuente. Consejo Privado de Competitividad \& CEPEC-Universidad del Rosario (2017). 
En el pilar de eficiencia de las empresas, las variables facilidad para abrir una empresa, facilidad para registrar propiedades y facilidad para obtener permisos de construcción fueron objeto de cambios metodológicos por parte del Doing Business, fuente principal de las mismas. Estas variables ahora se expresan a partir de su "distancia a la frontera", donde la frontera representa el mejor desempeño observado en todos los departamentos de la muestra.

Antioquia se mantiene en el segundo lugar en el pilar de desarrollo de mercados financieros y tercero en eficiencia de los mercados, por deba- jo de Bogotá en 2,45 puntos. Al igual que Bogotá, tiene el menor número de pagos de impuestos por año, obteniendo un puntaje de 10 en esta variable. Al mismo tiempo, en las variables de formalidad laboral, cobertura de seguros y saldo de cuentas de ahorro, el departamento ocupa el segundo lugar, estas cifras no son tan favorables para los departamentos de Meta y Magdalena, que ocupan puestos medios y bajos en este pilar.

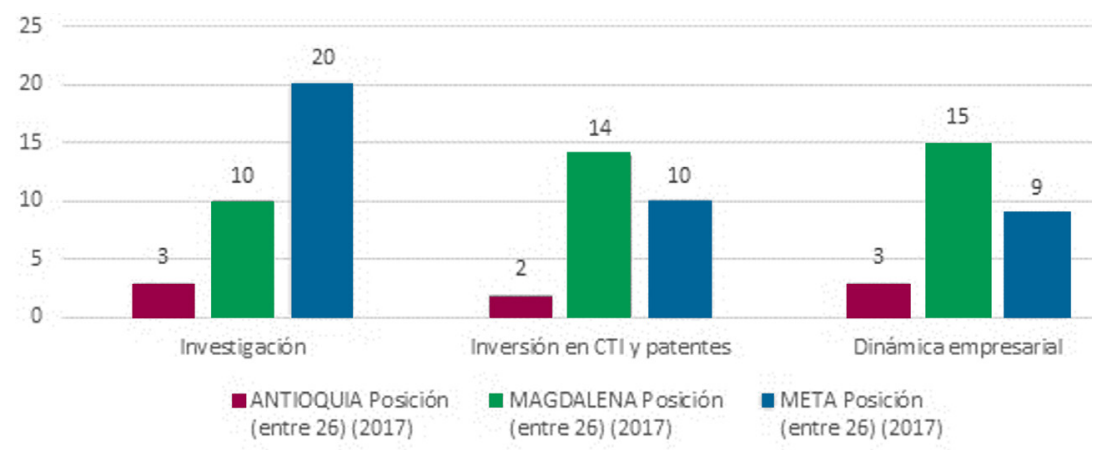

Gráfico 9. Indice comparativo innovación y dinamica empresarial departamentos de Antioquia, Magdalena y Meta. Fuente. Consejo Privado de Competitividad \& CEPEC-Universidad del Rosario (2017).

En 2017, para el pilar de la innovación y dinámica empresarial la región Cundinamarca/Bogotá ocupa la primera posición y conforma en sí misma el nivel extra líder. Le siguen como líderes: Antioquia, Santander, Risaralda, Caldas, Atlántico y Valle del Cauca. Se hallan en el nivel alto: Quindío, Boyacá y Bolívar. En el medio alto están: Tolima, San Andrés, Norte de Santander, Huila, Cesar y Meta.

En medio bajo están: Nariño, Magdalena, Cauca, Córdoba, Casanare y Sucre. Se hallan en el nivel bajo: Caquetá, la Guajira y Arauca. Le siguen como coleros: Putumayo, Amazonas, Chocó y Guaviare. Finalmente, son rezagados: Guainía, Vichada y Vaupés.

\section{Discusión}

\section{Ranking general}

El escalafón de la competitividad de los departamentos en estudio, destaca según la clasificación de (i) Extra líder, (ii) Líder, (iii) Alto, (iv) Medio alto, (v) Medio bajo, (vi) Bajo, (vii) Coleros y (viii) Coleros rezagados (CEPAL, 2017).

Los datos analizados muestran que, de los tres departamentos en estudio, Antioquia se cataloga como "Extra líder", porque es el único que mantiene su posición competitiva, en el 2 puesto, lo 
que lo identifica como un departamento ganador y estable.

Meta, clasificado como "Medio alto", porque, durante el período de análisis, muestra que tuvo una importante variación del ranking (16) al (11), razón por la cual se considera en el corto plazo como "ganador", en la medición del indicador.

En lo relativo, al departamento de Magdalena, clasificados como "Medio bajo", en mejora al pasar en el año 2016 del puesto 22 a 18 en 2017, en el corto plazo se reconocen como "emergente" y presentan tendencia a seguir mejorando su posición en los próximos años.

\section{Infraestructura productiva}

Las grandes desigualdades entre los departamentos en estudio en materia de infraestructura, muestra Antioquia con las mejores posiciones en servicios públicos, conectividad e infraestructura TIC, lo que la destaca ventajas competitivas ante Magdalena y Meta que tienen un marcado rezago en estos indicadores.

Dado que la infraestructura es el cuarto factor en importancia en el Indicador Global de Competitividad para las regiones, se requiere generar transferencia de buenas prácticas entre estos departamentos para que mediante alianzas los departamentos de Magdalena y Meta revisen el funcionamiento de Antioquia en infraestructura de servicios públicos, conectividad e infraestructura TIC, con lo que mejoren la inversión en los procesos en el corto plazo y mejoren su competitividad.

\section{Capital humano}

El indicador de capital humano, destaca como Meta supera en cobertura y calidad en educación básica y media al departamento de Antioquia y Magdalena.

\section{Conclusiones}

A partir de la investigación realizada, se puede concluir que:

En la medida, en que las regiones aprovechen al máximo el conocimiento, y los recursos de la región se puede generar nuevos modelos de desarrollo colaborativo público privado donde, el gobierno garantice el conocimiento como "propiedad pública para todos" de tal manera que sea accesible, se desarrollen capacidades para toda la población y determinar el rol de la tecnología donde el fin último es el desarrollo humano. Es decir, una sociedad más empoderada a partir del conocimiento científico y tecnológico.

El factor recurso humano se constituye en un indicador de competitividad fundamental por cuanto relaciona las características poblacionales diversas, de nivel de vida, cubrimiento de necesidades básicas, de empleo a nivel regional. Es hacia donde puedan converger las políticas públicas, y es lo que permite evaluar la efectividad de las políticas orientadas a beneficiar el recurso humano. Para ello es importante la creación, medición y seguimiento de indicadores: población, empleo, educación, capacitación y entrenamiento información pertinente que complementa el análisis realizado.

Las grandes desigualdades entre los departamentos en estudio en términos del índice de transparencia Meta y Antioquia superan al Magdalena mientras que en materia de gestión fiscal Antioquia y Magdalena superan al Meta, pero materia de seguridad y justicia Antioquia sobrepasa a Magdalena y Meta.

Otras grandes diferencias entre estos departamentos se dan en los pilares de bienestar e infraestructura, en este último se destacan servicios públicos, conectividad e infraestructura TIC, que se ubican como principales ventajas competitivas en las condiciones de vida, el capital humano y en la conectividad estratégica. Lo que señala las 
crecientes ventajas competitivas de la diversificación productiva del departamento de Antioquia que muestra unos resultados muy positivos, superiores a los departamentos de Magdalena y Meta.

Los contrastes en los niveles de corrupción que ostentan los departamentos de Magdalena y Meta, siguen por debajo de Antioquia, ya que este último prioriza inversiones desde las políticas públicas nacionales que deben fortalecerse orientarse en los departamentos de Magdalena y Meta principalmente para a apoyar las agendas de competitividad departamental.

Por último, se destaca la calidad en educación, fundamentalmente en las variables de calidad de docentes y cobertura de formación técnica y tecnológica, en las que Antioquia ocupa el primer y tercer lugar, igualmente sobresale en el segundo lugar en dominio de segundo idioma con una calificación, superando en estos pilares a Magdalena y Meta.

La relevancia de la integración de las regiones radica en los vínculos ya establecidos dentro de la región que pueden servir de base para generar un mayor desarrollo y lograr beneficios significativos.

\section{Referencias}

Canavire B., G., Carvajal O., L. C. (2017). Medellín y Antioquia: las cifras y las políticas. Recuperado en: https://razonpublica.com/index.php/ econom-y-sociedad-temas-29/10683-medell\%C3\%ADn-y-antioquia-las-cifras-y-las-pol\%C3\%ADticas.html

Cámara de Comercio de Medellín. (2017). Plan regional de competitividad: Recuperado en: http://www.sapiencia.gov.co/wp-content/ uploads/2017/08/plan-regional-competitividad-antioquia.pdf
Cámara de Comercio de Medellín. (2009). Avances de la estrategia cluster en Medellín y Antioquia. Recuperado en: https://www.camaramede1lin.com.co/site/Portals/0/Documentos/2016/ Cluster5.pdf

Cámara de Comercio de Santa Marta. (2017). Dinámica competitiva del Magdalena 2016-2017. Recuperado en: https://www.ccsm.org.co/ servicios-empresariales/servicios-ofertas-empresariales/estudios-economicos/investigaciones-e-indicadores-economicos/send/78-investigaciones/48207-dinamica-competitiva-del-magdalena-2016-2017.html

Consejo Privado de Competitividad \& Centro de Pensamiento en Estrategias Competitivas CEPEC - Universidad del Rosario. (2017). Índice departamental de competitividad 2017. Recuperado en: http://www.urosario.edu.co/competitividad/Documentos/CPC_IDC_2017-7-Nov. pdf.

Consejo Privado de Competitividad \& Centro de Pensamiento en Estrategias Competitivas CEPEC - Universidad del Rosario. (2016). Índice departamental de competitividad 2016. Recuperado en: http://www.urosario.edu.co/competitividad/contenido/Publicaciones/IDC-2016/

Consejo Privado de Competitividad (2017). Antioquia es la segunda región más competitiva del país, según el Índice Departamental de Competitividad 2017. Periódico NovaEtvetera, Recuperado en: http://www.urosario.edu.co/ Periodico-NovaEtVetera/Documentos/Competitividad/Antioquia_2017-VF.pdf

Consejo Privado de Competitividad (2017). Magdalena es la decimoctava región en el ranking del Índice Departamental de Competitividad 2017. Periódico NovaEtvetera, Recuperado en: http://www.urosario.edu.co/Periodico-NovaEtVetera/Documentos/Competitividad/ Magdalena_2017-VF.pdf

Consejo Privado de Competitividad (2017). Meta es la decimoprimera región en el ranking del 
Índice Departamental de Competitividad 2017. Periódico NovaEtvetera, Recuperado en: http://www.urosario.edu.co/Periodico-NovaEtVetera/Documentos/Competitividad/ Meta_2017-VF.pdf

Malamud, A. (2011). Conceptos, teorías y debates sobre la integración regional. Norteamérica, 6(2), 219-249. Recuperado en: http://www.scielo.org.mx/scielo.php?script=sci_arttext\&pi$\mathrm{d}=$ S1870-35502011000200008\&lng=es\&tlng=es.

Mincomercio. (5 de mayo de 2018). Perfiles económicos por departamento. Recuperado en: http:// www.mincit.gov.co/publicaciones/16724/perfiles_economicos_por_departamentos

Ramírez, J. \& De Aguas, J. (2017). Escalafón de la competitividad de los departamentos de Colombia 2017. CEPAL. Recuperado en: https://repositorio.cepal.org/bitstream/handle/11362/43156/1/S1800010_es.pdf

Rosales, O. (2014). Integración regional: hacia una estrategia de cadenas de valor inclusivas. CEPAL. LC/G.2594(SES.35/11). Recuperado en: https://repositorio.cepal.org/bitstream/ handle/11362/36733/S2014216_es.pdf?sequen$\mathrm{ce}=1 \&$ isAllowed $=\mathrm{y}$

Villaverde Castro, J. (s.f). Competitividad regional. Recuperado en: http://personales.unican.es/ villavej/documentos/prensa/2005/Competitividad.doc 